\title{
ALKALOIDS FROM LEAVES OF Annona muricata
}

\author{
S. Fofana, ${ }^{1}$ A. Keita, ${ }^{2}$ S. Balde, ${ }^{2}$ R. Ziyaev, ${ }^{1}$ and S. F. Aripova ${ }^{3}{ }^{*}$
}

UDC 547.944/945

We previously isolated from leaves of Annona muricata L. (Annonaceae) and characterized the alkaloids anonaine, xylopine, isolaureline, and coclaurine. In continuation of the study of alkaloids from leaves of $A$. muricata collected in the Republic of Guinea, we communicate the isolation from the phenolic and non-phenolic parts of total bases another five alkaloids (1-5).

Phenolic and non-phenolic fractions of total alkaloids were separated using chromatography over a column of silica gel. Alkaloids were eluted by benzene and $\mathrm{C}_{6} \mathrm{H}_{6}:$ EtOH in various ratios $(99: 1,98: 2,95: 5$, and 90:10). The separation was monitored by TLC analysis. Eluates containing pure compounds were combined and condensed. Crystals of alkaloids were isolated by recrystallization from benzene, acetone, and $\mathrm{CHCl}_{3}$ and by preparation of their salts. The pure alkaloids obtained in this manner were identified by chromatographic mobility, physical constants, and comparison of spectral data and with authentic samples.

Base 1, $\mathrm{C}_{18} \mathrm{H}_{21} \mathrm{NO}_{3}$, white crystals, mp $177-178^{\circ} \mathrm{C}$ (acetone), $[\alpha]_{\mathrm{D}}+25^{\circ}(\mathrm{EtOH})$. A mixed sample with authentic $N$-methylcoclaurine [2] did not depress the melting point.

Base 2, $\mathrm{C}_{17} \mathrm{H}_{17} \mathrm{NO}_{2}$, crystals obtained by crystallization from acetone, mp $175-176^{\circ} \mathrm{C},[\alpha]_{\mathrm{D}}-210^{\circ}\left(\mathrm{CHCl}_{3}\right)$. Comparison with an authentic sample identified it as asimilobin [3, 4].

Base 3, $\mathrm{C}_{19} \mathrm{H}_{21} \mathrm{NO}_{4}, \mathrm{mp} 125-126^{\circ} \mathrm{C}\left(\mathrm{C}_{6} \mathrm{H}_{6}\right)$, phenolic base, optically active, $[\alpha]_{\mathrm{D}}+42^{\circ}(c 0.2$, EtOH). UV spectrum $\left(\lambda_{\max }, \mathrm{EtOH}, \mathrm{nm}, \log \varepsilon\right): 220,280,313$ (4.50, 4.20, 4.18). Mass spectrum $(\mathrm{m} / \mathrm{z}): 327[\mathrm{M}]^{+}, 326(100)[\mathrm{M}-1]^{+}, 312[\mathrm{M}-15]^{+}$, $310[\mathrm{M}-17]^{+}, 296[\mathrm{M}-31]^{+}, 284[\mathrm{M}-43]^{+}, 269,253,163.5[\mathrm{M}]^{++}$. The data identified it as isoboldine [3, 5].

Base 4, non-phenolic, optically inactive, isolated as the hydrochloride of formula $\mathrm{C}_{18} \mathrm{H}_{17} \mathrm{NO}_{2} \cdot \mathrm{HCl}, \mathrm{mp} 263-265^{\circ} \mathrm{C}$ (dec.), identified as remerine [6,7].

Base 5, $\mathrm{C}_{17} \mathrm{H}_{9} \mathrm{NO}_{3}$, non-phenolic, optically inactive, high-melting crystalline base, yellow, $\mathrm{mp} 273-275^{\circ} \mathrm{C}\left(\mathrm{CHCl}_{3}\right)$. Comparison of data given above with the literature $[8,9]$ suggested that 5 was liriodenine. A mixed sample with an authentic sample of liriodenine did not depress the melting point. One spot was seen on TLC.

Thus, another five bases, benzyltetrahydroisoquinoline $N$-methylcoclaurine (1), aporphine asimilobin (2), remerine (3), isoboldine (4), and oxoaporphine liriodenine (5) were isolated from leaves of $A$. muricata and identified in addition to previously described alkaloids [1]. Alkaloids 2-5 were found for the first time in this plant species.

\section{REFERENCES}

1. S. Fofana, R. Ziyaev, A. Abdusamatov, and S. Kh. Zakirov, Khim. Prir. Soedin., 287 (2011).

2. R. Ziyaev, T. Irgashev, I. A. Israilov, N. D. Abdullaev, M. S. Yunusov, and S. Yu. Yunusov, Khim. Prir. Soedin., 239 (1977).

3. H. Guinaudeau, M. Leboeuf, and A. Cave, Lloydia, 38, 275 (1975); J. Nat. Prod., 42, 325 (1979); 46, 761 (1983); 51, 389 (1988).

4. M. Leboeuf, C. Legeueut, A. Cave, J. F. Desconclois, P. Forgacs, and H. Jacquemin, Planta Med., 42, 37 (1981).

5. R. Ziyaev, A. Abdusamatov, M. S. Yunusov, and S. Yu. Yunusov, Khim. Prir. Soedin., 81 (1991).

6. S. Rasamiwafy, R. Hocquemiller, H. Guinaudeau, A. Cave, and H. Jacquemin, J. Nat. Prod., 49, 1078 (1986).

7. R. Ziyaev, A. N. Arslanova, A. Abdusamatov, and S. Yu. Yunusov, Khim. Prir. Soedin., 518 (1986).

8. R. Ziyaev, A. Abdusamatov, and S. Yu. Yunusov, Khim. Prir. Soedin., 685 (1974).

9. $\quad$ A. Abdusamatov, R. Ziyaev, and S. Yu. Yunusov, Khim. Prir. Seodin., 112 (1974).

1) Centre Universitaire de Kindia, Republique de Guinee, e-mail: Rectorat@univ-kindia.org, ziyaev05@mail.ru; 2) Laboratoire de Chimie Organique de l'Universite de Conakry, Republique de Guinee; 3) S. Yu. Yunusov Institute of the Chemistry of Plant Substances, Academy of Sciences, Republic of Uzbekistan, Tashkent, fax: (99871) 120 64 75, e-mail: Salima_aripova@mail.ru. Translated from Khimiya Prirodnykh Soedinenii, No. 4, July-August, 2012, p. 637. Original article submitted December 5, 2011. 